\title{
Live imaging of developmental processes in a living meristem of Davidia involucrata (Nyssaceae)
}

\author{
Markus Jerominek ${ }^{1}{ }^{*}$, Kester Bull-Hereñ ${ }^{2,3}$, Melanie Arndt ${ }^{1}$ and Regine Claßen-Bockhoff ${ }^{1}$ \\ 1 Institut für Spezielle Botanik, Johannes Gutenberg-Universität, Mainz, Germany \\ ${ }^{2}$ Escuela de Pedagogía en Biología y Ciencias, Universidad Central de Chile, Santiago de Chile, Chile \\ ${ }^{3}$ Facultad de Ciencias Biológicas, Pontificia Universidad Católica de Chile, Santiago de Chile, Chile
}

Edited by:

Elena M. Kramer, Harvard

University, USA

Reviewed by:

Stewart Gillmor,

CINVESTAV-IPN, Mexico

Jacques Dumais, Universidad

Adolfo Ibáñez, Chile

\section{*Correspondence:}

Markus Jerominek, Institut für

Spezielle Botanik, Johannes

Gutenberg-Universität,

Anselm-Franz-von-Bentzel-Weg 9 a,

Mainz, 55099, Germany

e-mail: mail@spinningspecies.com
Morphogenesis in plants is usually reconstructed by scanning electron microscopy and histology of meristematic structures. These techniques are destructive and require many samples to obtain a consecutive series of states. Unfortunately, using this methodology the absolute timing of growth and complete relative initiation of organs remain obscure. To overcome this limitation, an in vivo observational method based on Epi-Illumination Light Microscopy (ELM) was developed and tested with a male inflorescence meristem (floral unit) of the handkerchief tree Davidia involucrata Baill. (Nyssaceae). We asked whether the most basal flowers of this floral unit arise in a basipetal sequence or, alternatively, are delayed in their development. The growing meristem was observed for 30 days, the longest live observation of a meristem achieved to date. The sequence of primordium initiation indicates a later initiation of the most basal flowers and not earlier or simultaneously as SEM images could suggest. $D$. involucrata exemplarily shows that live-ELM gives new insights into developmental processes of plants. In addition to morphogenetic questions such as the transition from vegetative to reproductive meristems or the absolute timing of ontogenetic processes, this method may also help to quantify cellular growth processes in the context of molecular physiology and developmental genetics studies.

Keywords: live imaging, in vivo, morphogenesis, floral unit meristem (FU meristem), epi-illumination light microscopy (ELM), Davidia involucrata, Nyssaceae

\section{INTRODUCTION}

One of the most significant technical advances of the last decades in plant sciences is the in vivo observation of developmental processes. In vivo techniques have the great advantage that they are non-destructive and allow imaging of phenomena as they occur within the plant body (Grandjean et al., 2004; Sijacic and Liu, 2010; Hiroi et al., 2013). Several innovations in light microscopy and fluorescence labeling technologies have offered amazing insights into developmental processes in meristematic tissues (Campilho et al., 2006; Reddy, 2008).

While these approaches primarily address gene expression or hormone flux issues (Grandjean et al., 2004; Heisler et al., 2005; Vernoux et al., 2011), traditional imaging techniques such as histology, scanning electron microscopy (SEM), epiillumination light microscopy (ELM) and computer tomography (CT, Staedler et al., 2013) have succeeded in providing clear information regarding morphogenesis at the tissue level. Unfortunately, these techniques are normally destructive and necessarily imply the observation of many individuals in different developmental states to reconstruct ontogenetic sequences. Thus, this approach demands some interpretation, since the same developing structure is not being observed among different samples.

Abbreviations: SEM, scanning electron microscopy; ELM, epi-illumination light microscopy; FU, floral unit.
Particularly, this can become a complex issue when reconstructing the development of numerically variable structures, e.g., condensed inflorescences known as "floral units" (Claßen-Bockhoff and Bull-Hereñu, 2013). In floral units (FU), flower primordia usually fractionate in either a centripetal [e.g., umbellets in Apiaceae, (Bull-Hereñu and Claßen-Bockhoff, 2010)] or centrifugal sequence [e.g., cyathia in Euphorbia, (Prenner and Rudall, 2007)] (Figure 1A). If primordia appear almost simultaneously the sequence is interpreted from the arrangement and size of flower primordia, assuming that the smaller ones have been initiated later (Figure 1A). A direct size-age correlation is used because of the a priori assumption that all flower primordia share similar growth rates. However, simultaneous initiation of primordia but a slower growth rate in the basal primordia would create a false impression of centrifugal initiation (Figure 1B).

This is a case where traditional imaging techniques find their interpretational limit when trying to elucidate which ontogenetic process is actually occurring.

The floral unit of Davidia involucrata Bail. (Nyssaceae, Figure 1F) illustrates this conflict. During development, a number of flower primordia arise almost simultaneously on the FU meristem (Figure 1C); those in the equatorial zone are larger than the most basal ones (Figures 1D,E). As stated above it remains unclear whether the basal flower primordia in D. involucrata 

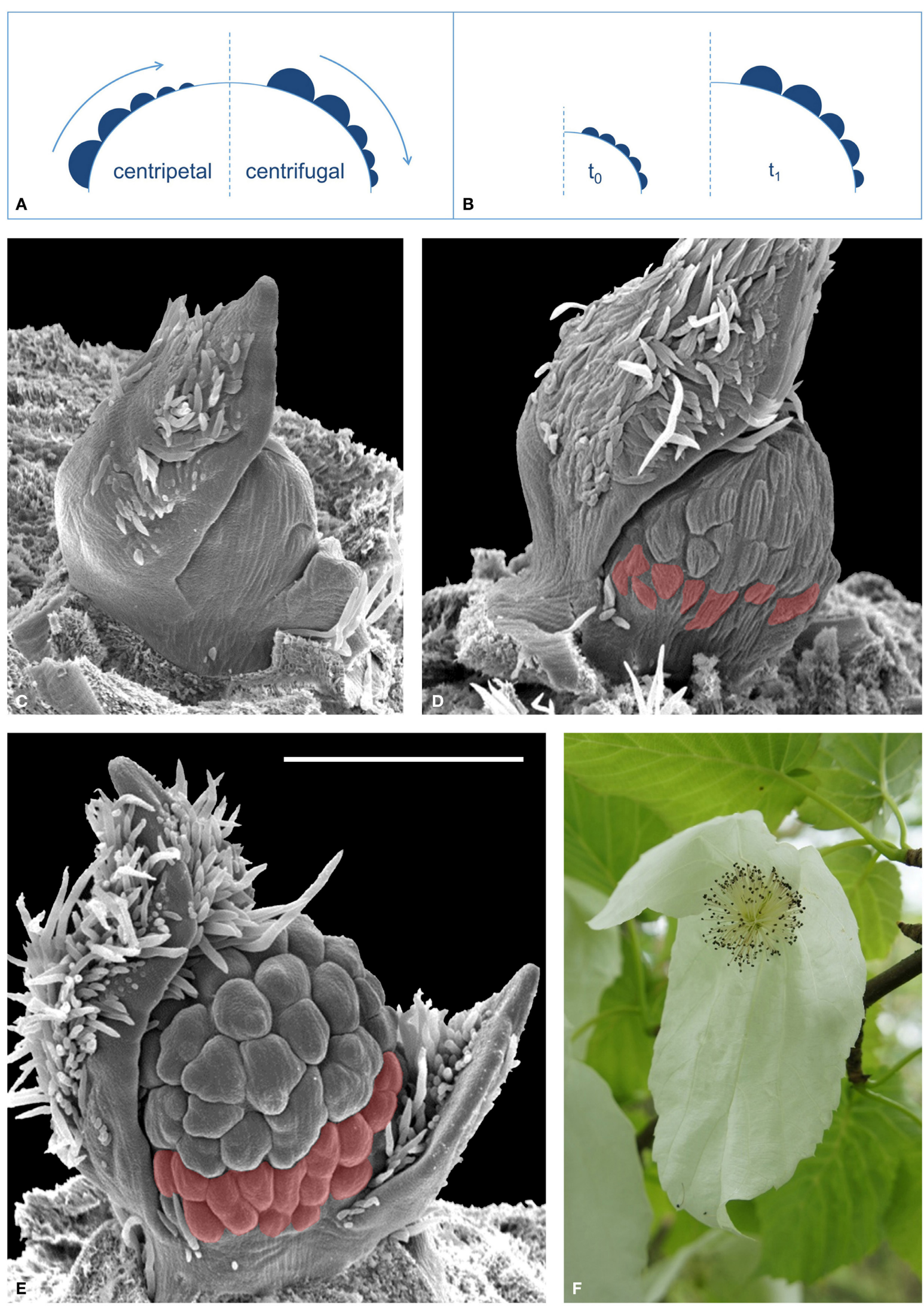

FIGURE 1 | Continued 


\section{FIGURE 1 | Continued}

Development of reproductive meristems. (A) The relative size of flower primordia (filled semicircles) of a reproductive meristem (large dome) is generally used to reconstruct the sequence of initiation (arrow), which either occurs toward the center, i.e., "centripetal" (left sketch), or toward the flanks, i.e., "centrifugal" (right sketch). (B) Different growing rates of simultaneously initiated flower primordia lead to a false interpretation of the initiation sequence (here centrifugal). (C-E), SEM images of a developing FU of Davidia involucrata: (C) Young meristem starting to fractionate flower primordia. (D) Later stage, with many flower primordia initiated. Note that the most basal primordia (red) are smaller. (E) A male FU meristem with stamen initiation in equatorial primorida and delayed development of the most basal flowers (red). All SEM images are in the same scale, bar line $=500 \mu \mathrm{m}$. (F) Male inflorescence (FU) of $D$. involucrata subtended by two conspicuous extrafloral bracts. originate later, i.e., in a centrifugal sequence, or grow more slowly (Figure 1B). This problem can only be solved by in vivo observation of the same growing meristem. By developing an automated ELM live imaging technique it was possible to record the development of a male floral unit meristem of $D$. involucrata for a period of 30 days.

\section{MATERIALS AND METHODS PLANT MATERIAL}

A FU meristem of $D$. involucrata was taken from a tree cultivated in the Botanical Garden at the University of Mainz, Germany. The meristem, located at the tip of a short branch, was dissected and mounted with styrofoam in a histological staining dish while the site of fracture was submerged in tap water (Figure 2). To prevent dehydration the meristem was directly covered by a modified probe tube $(50 \mathrm{ml})$ that was used as moisture chamber. The lower side was closed by the water of the staining dish and the upper side was covered with a latex membrane that enveloped the objective lens. During observation, the water in the staining dish was checked and refilled twice a week.

\section{EPI-ILLUMINATION LIGHT MICROSCOPY (ELM)}

Since common binocular microscopes have angular optical paths that produce a shift of images in a stack, we used a monocular microscope (Leitz Wetzlar, Figure 2). Photographs were taken with a Canon Powershot G9 that was mounted on the eyepiece and triggered by a computer (Canon CameraWindow). For an automated stacking procedure a stepper motor was adapted to the focus wheel of the microscope and controlled by a fourchannel motor driver circuit (L298N). For illumination a LED light was switched on during the image capture process. The whole stacking cycle was controlled by an open-source microcontroller (Arduino Leonardo) that was connected to the motor driver to change the focus. The camera was triggered by an implemented keyboard source code via a computer and the LED was controlled via a relay. Each cycle began with the activation of the LED and was followed by raising the microscope to the highest focus point. The next two steps that triggered the camera and moved the microscope to a lower focus position were repeated until the lowest focus point was reached (70 times). To finish the cycle the LED was turned off. For live imaging the cycle was triggered and repeated once per hour by a timer (remote control for cameras). Since the meristem grew, the focus range was checked twice a week and manually readjusted. After the meristem became too large the magnification was reduced from a 10 -fold to a 4 -fold objective (comp. Figures $3 \mathrm{~A}-\mathrm{F}$ and G-L). Accordingly, live observation is divided into two time lapse videos.

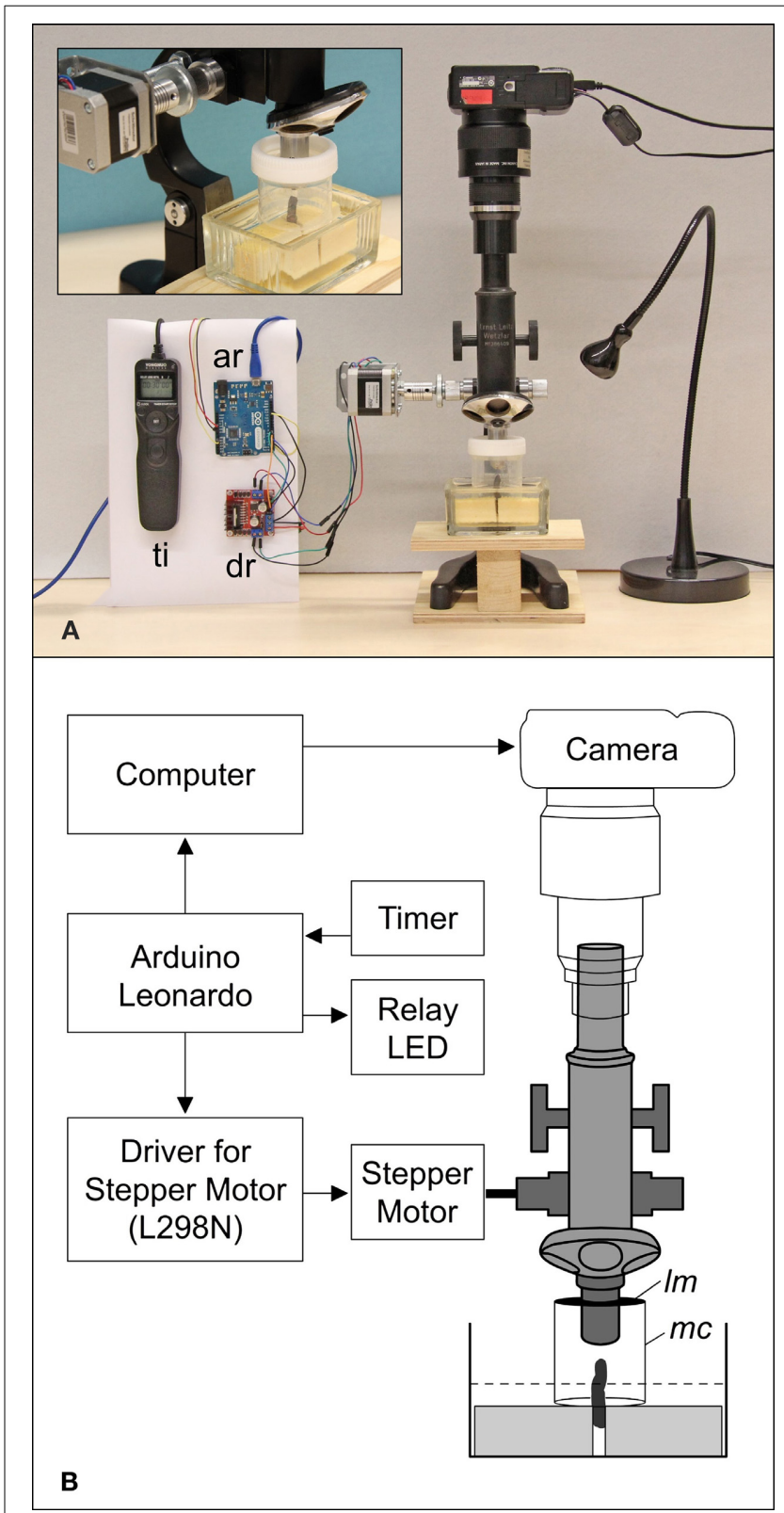

FIGURE 2 | Setup (A) and schematic diagram (B) of the automated arrangement. A branch of Davidia involucrata is placed in a staining dish with tap water and fixed with two styrofoam blocks (A, Close-up). The tip of the branch (meristem) is protected by a modified probe tube forming a moisture chamber $(\mathrm{mc})$. The upper side is covered by a latex membrane $(\mathrm{Im})$ that envelops the objective lens. The lower side is closed by the water level (dotted line). ar, Arduino Leonardo (microcontroller); dr, driver for stepper motor; Im, latex membrane; mc, moisture chamber; ti, timer remote control. 

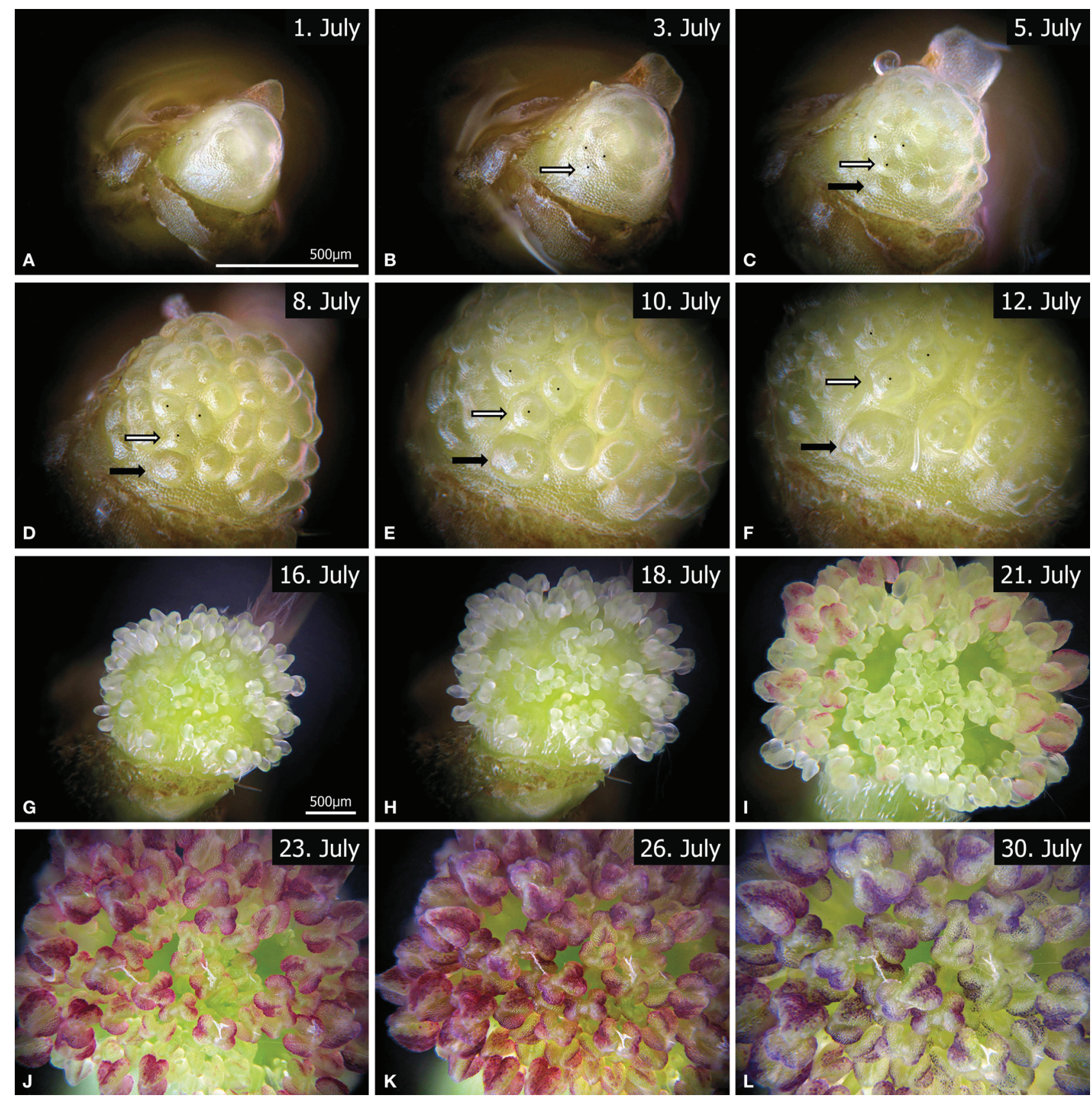

FIGURE 3 | Twelve representative images from the time-lapse videos (Video S1, S2), showing the development of a male FU, from flower (A-D) and stamen initiation (EF) to anther development (G-K). Due to meristem growth the magnification was changed between (A-F) (Video S1) and (G-L)
(Video S2). The corresponding bar lines are indicated in $(\mathbf{A}, \mathbf{G})$. The white and black arrows mark two individual primordia at different developmental states. The outer primordia (black arrow) are clearly initiated later than the inner ones (white arrow). Videos available at www.spinningspecies.com/davidia.html.

\section{IMAGE PROCESSING}

To generate a time-lapse video all images from each stack had to be combined to one single sharp image (Wilson et al., 2006). This image processing was performed with CombineZP (http:// www.hadleyweb.pwp.blueyonder.co.uk/), which allows automation of this process for all image stacks (CZBatch.exe). Here the soft stack algorithm was applied. To compensate the shift of photographs due to image processing as well as to the readjustments of the focus range, all images were arranged by adjusting and shifting them consecutively with Amira (Mercury Computer Systems). The resulting time-lapse videos were generated either with JPGVideo (http://www.ndrw.co.uk/) or by loading all images into a flash movie (Adobe Flash CS3, www. spinningspecies.com/davidia.html). 


\section{RESULTS AND DISCUSSION MERISTEM DEVELOPMENT}

The observation began with an undifferentiated meristem (Figure 3A, Video S1). Flower initiation was clearly seen on the 3rd day of observation (Figure 3B), when almost all flower primordia appeared more or less simultaneously. After 2 days and a considerable enlargement of the FU meristem, additional primordia arose at its base (Figure 3C, black arrow). In contrast to the SEM sequence (Figures 1C,D) the most basal primordia develop faster, since they became larger than the ones in the equatorial zone as the FU meristem continued to expand during the next 5 days (Figures 3D,E). At day 12 the first anther primordia could be observed in the basal flowers (Figure 3F). These anthers developed faster than those of the upper flowers (Figures 3G-I, Video S2). Finally, the anthers covered the whole meristem and became red (Figures 3J,K) to purple (Figure 3L).

The observation of the male FU of $D$. involucrata performed here indicates that the smaller size of the proximal flower primordia may be due to basipetal initiation. The initiation of ray florets in Asteraceae heads exhibits a similar phenomenon; SEM studies suggested that they emerged later (Harris et al., 1991; Harris, 1995; Bello et al., 2013). However, the alternative "basal delay" hypothesis discussed here cannot be rejected until an in vivo observation of a head meristem is performed.

\section{LIVE IMAGING WITH ELM}

Our study shows that ELM can be used to obtain detailed structural information of a growing meristem. This technique could be an alternative to replica molds (Williams and Green, 1988; Green et al., 1991; Dumais and Kwiatkowska, 2001; Kwiatkowska, 2006; Barabé et al., 2007; Szczęsny et al., 2009) and confocal microscopy (Grandjean et al., 2004; Heisler et al., 2005; Campilho et al., 2006; Reddy, 2008; Sijacic and Liu, 2010; Vernoux et al., 2011), the only imaging techniques that have been successful in obtaining data from the development of a single meristem up to now. In contrast to conventional methods, in vivo techniques are able to analyze developmental timing and clear up relative initiation issues (Table 1). While SEM only allows reconstruction of developmental steps, live imaging reveals the absolute time of organ initiation and duration of growth. Moreover, this low-cost method is compatible with color imaging and is simple: objects do not need to be fixed, critical point dried or sputtered.

Although ELM has been successfully used for fixed material (Bartlett et al., 2008; Dadpour et al., 2008, 2011, 2012; Oraei et al., 2013), live ELM imaging of a developing meristem has been largely neglected in the past. The main reason may have been the difficulty in maintaining the tissue healthy for several days.

The spatial resolution of ELM depends on the properties of the microscope and the stepper motor. Thereby the depth of field depends on the aperture of the objective for a single image and on the range of the fine focus for the whole stack. The distance between single images depends on the physical resolution of the stepper motor. We used 200 steps per revolution which provided a resolution of $1 \mu \mathrm{m}$, but this could be made smaller by driving the motor with micro-steps.

The temporal resolution of the system is defined by the duration of a stack, which depends on the speed of the motor
Table 1 | Comparison of different visualizing techniques used in plant development.

\begin{tabular}{ll}
\hline Technique & Pros and Cons \\
\hline Histology & + Reveals inner structures \\
& + Reveals gene expression patterns \\
& - Destructive \\
\hline SEM & + Retrieves high structural details \\
& - Destructive \\
\hline Confocal microscopy & + In vivo observation \\
& + Reveals gene expression patterns \\
& - High cost \\
& - Demands appropriate culturing methods \\
& - Fluorescent labeling required \\
& + In vivo observation \\
& + Retrieves high structural details \\
& - Time-consuming and limited application \\
\hline Replica molds & + Reveals inner structures \\
& - High cost and time-consuming \\
& - Destructive \\
\hline Micro-CT & + Inexpensive \\
& + Color imaging \\
& - Destructive \\
& + Inexpensive \\
& + Color imaging \\
& + In vivo observation \\
\hline LLM & \\
& \\
& \\
&
\end{tabular}

and the exposure time of the camera. Since we used a long exposure time ( $1 \mathrm{~s})$ and a low motor speed, the stacking procedure took about $15 \mathrm{~min}$. Another serious problem was the correct illumination of the object. To avoid reflections that would hamper the stacking procedure we used a weak indirect illumination of the object with long exposure times. Therefore, the light cone was directed to the upper latex membrane (Figure 3B, lm).

\section{CONCLUSIONS}

By means of this automated live ELM system it is possible to create real-time high resolution records of growing plant meristems. The methodology illustrated here can contribute substantially to ontogenetic plant imaging, as it provides a potential easyto-operate tool for monitoring and quantification of growth processes. On the morphogenetic level it can give new insights into organ growth, meristematic transitions, absolute timing of plant development and morphometric responses to experimental stimuli (Reinhardt et al., 2003; Bello et al., 2013; Claßen-Bockhoff and Bull-Hereñu, 2013). On the cellular level it may help to analyze cell expansion and division in the context of molecular physiology and developmental genetic studies (Hamant et al., 2010; Sijacic and Liu, 2010; Uyttewaal et al., 2012; Sassi and Vernoux, 2013). 


\section{AUTHOR CONTRIBUTIONS}

Markus Jerominek and Kester Bull-Hereñu initiated the study. Markus Jerominek designed the experimental setup and drafted the manuscript. Melanie Arndt provided the SEM data of FU development in Davidia involucrata, supervised by Regine Claßen-Bockhoff. Kester Bull-Hereñu and Regine ClaßenBockhoff analyzed the data and contributed to the final manuscript.

\section{ACKNOWLEDGMENTS}

We are grateful for helpful comments and suggestions provided by the referees.

\section{SUPPLEMENTARY MATERIAL}

The Supplementary Material for this article can be found online at: http://www.frontiersin.org/journal/10.3389/fpls.2014. 00613/abstract

\section{REFERENCES}

Barabé, D., Lacroix, C., and Jeune, B. (2007). Following the initiation and development of individual leaf primordia at the level of the shoot apical meristem: the case of distichous phyllotaxis in Begonia. Ann. Bot. 99, 555-560. doi: $10.1093 / \mathrm{aob} / \mathrm{mcl} 286$

Bartlett, M., Kirchoff, B., and Specht, C. (2008). Epi-illumination microscopy coupled to in situ hybridization and its utility in the study of evolution and development in non-model species. Dev. Genes Evol. 218, 273-279. doi: 10.1007/s00427-008-0211-6

Bello, M. A., Álvarez, I., Torices, R., and Fuertes-Aguilar, J. (2013). Floral development and evolution of capitulum structure in Anacyclus (Anthemideae, Asteraceae). Ann. Bot. 112, 1597-1612. doi: 10.1093/aob/mcs301

Bull-Hereñu, K., and Claßen-Bockhoff, R. (2010). Developmental conditions for terminal flower production in apioid umbellets. Plant Div. Evol. 128, 221-232. doi: 10.1127/1869-6155/2010/0128-0010

Campilho, A., Garcia, B., Toorn, H. V. D., Wijk, H. V., Campilho, A., and Scheres, B. (2006). Time-lapse analysis of stem-cell divisions in the Arabidopsis thaliana root meristem. Plant J. 48, 619-627. doi: 10.1111/j.1365-313X.2006.02892.x

Claßen-Bockhoff, R., and Bull-Hereñu, K. (2013). Towards an ontogenetic understanding of inflorescence diversity. Ann. Bot. 112, 1523-1542. doi: $10.1093 / \mathrm{aob} / \mathrm{mct} 009$

Dadpour, M. R., Grigorian, W., Nazemieh, A., and Valizadeh, M. (2008). Application of epi-illumination light microscopy for study of floral ontogeny in fruit trees. Int. J. Bot. 4, 49-55. doi: 10.3923/ijb.2008.49.55

Dadpour, M. R., Naghiloo, S., and Gohari, G. (2011). Inflorescence and floral ontogeny in Osteospermum ecklonis (Asteraceae). Botany 89, 605-614. doi: 10.1139/b11-052

Dadpour, M. R., Naghiloo, S., and Neycharan, S. F. (2012). The development of pistillate and perfect florets in Xeranthemum squarrosum (Asteraceae). Plant Biol. 14, 234-243. doi: 10.1111/j.1438-8677.2011.00469.x

Dumais, J., and Kwiatkowska, D. (2001). Analysis of surface growth in shoot apices. Plant J. 31, 229-241. doi: 10.1046/j.1365-313X.2001.01350.x

Grandjean, O., Vernoux, T., Laufs, P., Belcram, K., Mizukami, Y., and Traas, J. (2004). In vivo analysis of cell division, cell growth, and differentiation at the shoot apical meristem in Arabidopsis. Plant Cell 16, 74-87. doi: 10.1105/tpc.017962

Green, P., Havelange, A., and Bernier, G. (1991). Floral morphogenesis in Anagallis: scanning-electron-micrograph sequences from individual growing meristems before, during, and after the transition to flowering. Planta 185, 502-512. doi: 10.1007/BF00202959

Hamant, O., Traas, J., and Boudaoud, A. (2010). Regulation of shape and patterning in plant development. Curr. Opin. Genet. Dev. 20, 454-459. doi: 10.1016/j.gde.2010.04.009

Harris, E. (1995). Inflorescence and floral ontogeny in Asteraceae: a synthesis of historical and current concepts. Bot. Rev. 61, 93-278. doi: 10.1007/BF02887192
Harris, E. M., Tucker, S. C., and Urbatsch, L. E. (1991). Floral initiation and early development in Erigeron philadelphicus (Asteraceae). Am. J. Bot. 78, 108-121. doi: $10.2307 / 2445234$

Heisler, M. G., Ohno, C., Das, P., Sieber, P., Reddy, G. V., Long, J. A., et al. (2005). Patterns of auxin transport and gene expression during primordium development revealed by live imaging of the Arabidopsis inflorescence meristem. Curr. Biol. 15, 1899-1911. doi: 10.1016/j.cub.2005.09.052

Hiroi, K., Sone, M., Sakazono, S., Osaka, M., Masuko-Suzuki, H., Matsuda, T., et al. (2013). Time-lapse imaging of self- and cross-pollinations in Brassica rapa. Ann. Bot. 112, 115-122. doi: 10.1093/aob/mct102

Kwiatkowska, D. (2006). Flower primordium formation at the Arabidopsis shoot apex: quantitative analysis of surface geometry and growth. J. Exp. Bot. 57, 571-580. doi: 10.1093/jxb/erj042

Oraei, M., Gohari, G., Esmaillou, Z., and Naghiloo, S. (2013). Comparative ontogeny of perfect and pistillate florets in Senecio vernalis (Asteraceae). Flora 208, 285-292. doi: 10.1016/j.flora.2013.04.002

Prenner, G., and Rudall, P. J. (2007). Comparative ontogeny of the cyathium in Euphorbia (Euphorbiaceae) and its allies: exploring the organ-flowerinflorescence boundary. Am. J. Bot. 94, 1612-1629. doi: 10.3732/ajb.94.10.1612

Reddy, G. V. (2008). Live-imaging stem-cell homeostasis in the Arabidopsis shoot apex. Curr. Opin. Plant Biol. 11, 88-93. doi: 10.1016/j.pbi.2007.10.012

Reinhardt, D., Frenz, M., Mandel, T., and Kuhlemeier, C. (2003). Microsurgical and laser ablation analysis of interactions between the zones and layers of the tomato shoot apical meristem. Development 130, 4073-4083. doi: 10.1242/dev. 00596

Sassi, M., and Vernoux, T. (2013). Auxin and self-organization at the shoot apical meristem. J. Exp. Bot. 64, 2579-2592. doi: 10.1093/jxb/ert101

Sijacic, P., and Liu, Z. (2010). Novel insights from live-imaging in shoot meristem development. J. Integr. Plant Biol. 52, 393-399. doi: 10.1111/j.17447909.2010.00941.x

Staedler, Y. M., Masson, D., and Schönenberger, J. (2013). Plant tissues in 3D via $\mathrm{X}$-ray tomography: simple contrasting methods allow high resolution imaging. PLoS ONE 8:e75295. doi: 10.1371/journal.pone.0075295

Szczęsny, T., Routier-Kierzkowska, A.-L., and Kwiatkowska, D. (2009). Influence of clavata3-2 mutation on early flower development in Arabidopsis thaliana: quantitative analysis of changing geometry. J. Exp. Bot. 60, 679-695. doi: 10.1093/jxb/ern312

Uyttewaal, M., Burian, A., Alim, K., Landrein, B., Borowska-Wykret, D., Dedieu, A., et al. (2012). Mechanical stress acts via katanin to amplify differences in growth rate between adjacent cells in Arabidopsis. Cell 149, 439-451. doi: 10.1016/j.cell.2012.02.048

Vernoux, T., Brunoud, G., Farcot, E., Morin, V., Van Den Daele, H., Legrand, J., et al. (2011). The auxin signalling network translates dynamic input into robust patterning at the shoot apex. Mol. Syst. Biol. 7, 508. doi: 10.1038/msb.2011.39

Williams, M. H., and Green, P. B. (1988). Sequential scanning electron microscopy of a growing plant meristem. Protoplasma 147, 77-79. doi: 10.1007/bf01403879

Wilson, T. C., Charlton, W. A., and Posluszny, U. (2006). Increased image depth for epi-illumination microscopy. Can. J. Bot. 84, 508-513. doi: 10.1139/b06-018

Conflict of Interest Statement: The authors declare that the research was conducted in the absence of any commercial or financial relationships that could be construed as a potential conflict of interest.

Received: 13 August 2014; accepted: 20 October 2014; published online: 13 November 2014.

Citation: Jerominek M, Bull-Hereñu K, Arndt M and Claßen-Bockhoff R (2014) Live imaging of developmental processes in a living meristem of Davidia involucrata (Nyssaceae). Front. Plant Sci. 5:613. doi: 10.3389/fpls.2014.00613

This article was submitted to Plant Evolution and Development, a section of the journal Frontiers in Plant Science.

Copyright () 2014 Jerominek, Bull-Hereñu, Arndt and Claßen-Bockhoff. This is an open-access article distributed under the terms of the Creative Commons Attribution License (CC BY). The use, distribution or reproduction in other forums is permitted, provided the original author(s) or licensor are credited and that the original publication in this journal is cited, in accordance with accepted academic practice. No use, distribution or reproduction is permitted which does not comply with these terms. 